\title{
Residual effects of eszopiclone and placebo in healthy elderly subjects: a randomized double-blind study
}

\author{
Jun Takahashi ${ }^{1}$ - Takashi Kanbayashi ${ }^{1,2}$ - Sachiko Ito Uemura ${ }^{3}$ - Youhei Sagawa ${ }^{1}$ • \\ Kou Tsutsui $^{1} \cdot$ Yuya Takahashi $^{1} \cdot$ Yuki Omori $^{1}$ - Aya Imanishi ${ }^{1} \cdot$ Masahiro Takeshima $^{1}$. \\ Masahiro Satake $^{3} \cdot$ Tetsuo Shimizu $^{1,2}$
}

Received: 12 December 2016/Accepted: 6 May 2017/Published online: 19 May 2017

(c) The Author(s) 2017. This article is an open access publication

\begin{abstract}
Next-day residual effects are a common problem with current hypnotics. The purpose of the present study was to evaluate the residual effects of eszopiclone on the physical and cognitive functions of healthy elderly people in the early morning and the day following drug administration. Four men and six women aged 63-72 years were administered eszopiclone $1 \mathrm{mg}$ or placebo in a randomized, double-blind and crossover design. Measures of objective parameters and subjective ratings were obtained at 4:00, 6:00, and every $2 \mathrm{~h}$ from 6:00 to 16:00 hours. For the timed up-and-go test, the main effects of time were seen. For the critical flicker fusion, eszopiclone had significantly worse results compared to placebo in early morning (4:00). There were no significant differences between eszopiclone and placebo in other objective assessments. For the sleep latency, eszopiclone had significantly shorter results compared to placebo (eszopiclone vs placebo $=28.4$ vs $52.5 \mathrm{~min}, p=0.047$ ). Feeling of deep sleep and the number of wake after sleep onset did not show any significant differences between eszopiclone and placebo. Based on the above results, the changes of physical and cognitive functions in the healthy elderly after taking hypnotics, it was found that eszopiclone $1 \mathrm{mg}$ is likely to be unharmful for the healthy elderly. Further studies of elderly insomniacs with midnight awakenings are needed.
\end{abstract}

Jun Takahashi

june.takahashi0301@gmail.com

1 Department of Psychiatry, Akita University Graduate School of Medicine, Akita, Japan

2 International Institute for Integrative Sleep Medicine (WPIIIIS), University of Tsukuba, Tsukuba, Japan

3 Department of Physical Therapy, Akita University Graduate School of Health Sciences, Akita, Japan
Keywords Hypnotics - Residual effects - Benzodiazepine · Critical flicker fusion test $\cdot$ Eszopiclone

\section{Introduction}

Previous studies have reported that the prevalence of insomnia is $8-18 \%$, and females are more prevalent than males $[1,2]$. In Japan, the prevalence of insomnia is $17.3 \%$ in males and $21.5 \%$ in females [3-5]. The occurrence of insomnia symptoms appeared to be associated with advancing age.

Although national panels have long cautioned against the use of hypnotics in the management of chronic insomnia [6], the use rate of hypnotics of elderly people is about $10-15 \%$ which is about 5 times that of young adults [7] and prescription of hypnotics is gradually increasing every year [8].

Hypnotics including benzodiazepine (BZ) relatively affect the physical and cognitive functions the next day. The association between the residual effects of hypnotics and road traffic accidents is often researched in Western countries, and it has been reported that the use of benzodiazepine hypnotics increases the odds ratio of traffic accident risk up to 1.19 [9-11].

In the elderly, the sensitivity to the drugs increases and blood concentration tends to rise because metabolic capacity declines. For this reason, the association between the use of benzodiazepine hypnotics in the elderly and the risk of falls and fracture is well established, and the use of hypnotics is often discussed as a public health problem $[12,13]$.

Some research evaluated the effects of hypnotic on the physical and cognitive functions in healthy adults [14, 15], but research on the elderly is few $[16,17]$. 
Uemura et al. reported a significant difference in some functions between the use of hypnotics and placebo in the healthy elderly [16]. However, there are still few studies on the use of hypnotics in the elderly, whose physical and cognitive impairments depend on aging.

There are some studies that use hypnotics in daytime, but quite few studies use hypnotics before sleep and evaluate their effects during the night $[16,17]$. According to a regional survey in Japan in 2008, brotizolam, zolpidem, and rilmazafone are the top three of the most prescribed hypnotics [18]. The common feature of these hypnotics is the short half-life effects.

These short-acting hypnotics are considered to have no residual effect after 8 hours of sleep. Since, mid-arousal by urination in the elderly increases, the total sleep time tends to be short. Its evaluation for functions about $5 \mathrm{~h}$ after oral administration is considered to be more practical.

Eszopiclone, which is classified as a non-BZ that acts on GABA/BZ receptor chloride channel complex, is recently available in Japan. Non-BZ agents are much more selective for the $\mathrm{BZ}$ receptor subtypes $\left(\mathrm{GABA}_{\mathrm{A}}\right)$ and have reportedly fewer side effects $[19,20]$.

We evaluated the differences of the elderly's physical and cognitive functions and subjective assessments using eszopiclone at night by randomized and double-blinded trials.

\section{Methods}

\section{Design}

The study was a randomized, double-blind, active (eszopiclone $1 \mathrm{mg}$ ) and placebo-controlled, two-period crossover study. Akita University Ethics Committee approved the protocol. The study was carried out in accordance with the principles based on the Declaration of Helsinki, and written informed consent was obtained from all subjects.

\section{Subjects}

4 men and 6 women aged 63-72 years and who were all healthy were eligible to enroll if they had a usual bedtime between 20:00 and 24:00 hours. The subjects agreed to avoid exercise that was strenuous or that they were unaccustomed to during the study. They were required to be in good health, as confirmed by their medical history, physical examination, and laboratory tests (hematology and clinical biochemistry). Exclusion criteria included the use of hypnotics within the previous 1 year, a history of drug or alcohol abuse and of repeated falls or a fracture secondary to a fall within the past 2 years. Subjects were required to abstain from prescription and nonprescription drugs and supplements. During the study, subjects were required to limit their daily consumption of alcoholic beverages containing less than $30 \mathrm{~g}$ alcohol per day. Alcoholic beverages were not permitted from $48 \mathrm{~h}$ before admission to the unit or before pre-study and post-study visits. Both caffeine and nicotine were prohibited $24 \mathrm{~h}$ before each visit to the experimental room set up at a local hotel.

\section{Procedure}

Subjects were randomized to treatment sequences including eszopiclone $1 \mathrm{mg}$ and placebo administered in a crossover fashion, with a 6-day washout period between each treatment. Hypnotics or placebo were orally administered to each subject at bedtime (23:00) and lights were then turned off. On the morning following dosing, lights were turned on at 4:00 and 06:00 (5 and $7 \mathrm{~h}$ post-dosing) (Fig. 1). Measurement of objective parameters and subjective parameters were obtained beginning at 4:00 $(5 \mathrm{~h}$ post-dosing) on Day 2. Subjects remained in the experimental room and close to bedrooms from early in the evening until $17 \mathrm{~h}$ post-dosing. All subjects took the same breakfast.

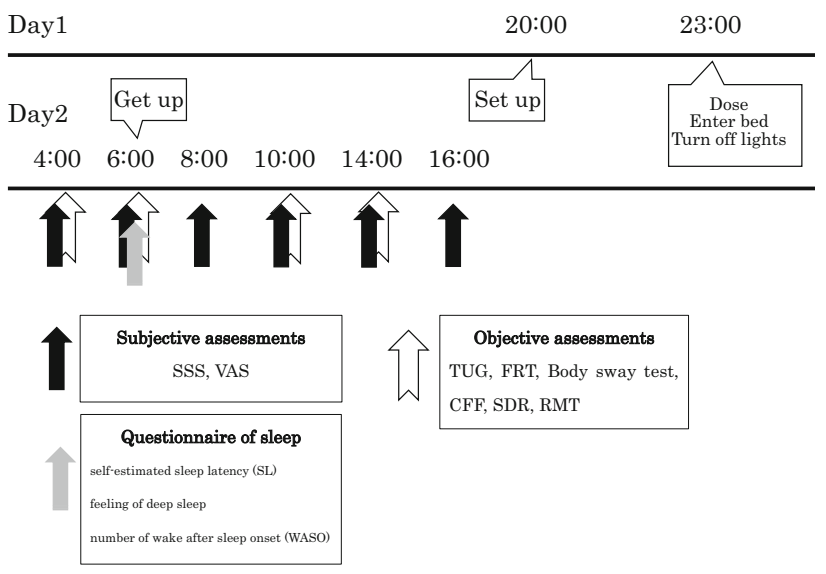

Fig. 1 Procedure. Eszopiclone or placebo was orally given to each subject at bedtime (23:00 hours, Day 1) and lights were turned off. On the morning following dosing, lights were turned on at 4:00 and 6:00 hours ( 5 and $7 \mathrm{~h}$ post-dose) on Day 2. Measurement of subjective parameters was obtained at 4:00, 6:00, 8:00, 10:00, 14:00 hours, and 16:00 hours on Day 2. Questionnaire on sleep was obtained at 6:00 hour on Day 2. Objective parameters were obtained at 4:00, 6:00, 10:00, and 14:00 hours on Day 2. Subjects remained in the hotel from early on the evening of day 1 until 16:00 hour on day 2. SSS Stanford Sleepiness Scale, VAS visual analog scale, Questionnaires of sleep questionnaires of self-estimated sleep latency (SL), feeling of deep sleep number of wake after sleep onset (WASO), CFF critical flicker fusion, FRT functional reach test, $T U G$ the timed upand-go test, $S D R$ simple discriminatory reaction, STM short-term memory test 


\section{Objective assessments}

\section{Physical functions}

The timed up-and-go (TUG) test The TUG test was performed according to the method described by Podsiadlo and Richardson [21]. Participants had to stand up from a sitting position (height of chair $=40 \mathrm{~cm}$ ) and walk $3 \mathrm{~m}$ along a line, perform a $180^{\circ}$ turn, and walk back to the chair and sit down; this was timed. The TUG tests were conducted twice, and the best time (where appropriate) was used. Smaller values were better results for TUG.

Functional reach test (FRT) In the FRT, the protocol described by Duncan et al. [22] was applied and a GB$200^{\circledR}$ (OG giken, Okayama, Japan) was used. Each participant was positioned with one arm raised at $90^{\circ}$ and fingers extended. A yardstick was mounted on the wall at shoulder height. The distance that a participant could reach while extending forward from the initial upright posture to the maximal anterior leaning posture without moving or lifting the feet was visually measured in $\mathrm{cm}$, according to where the middle fingertip was positioned on the mounted yardstick. The distances were measured for two attempts, and these were averaged to obtain the FR score. Larger values were better results for FRT.

Body sway test Body sway test $(\mathrm{cm})$ reflects the standing balance using a stabilometric platform [Zebris WinFDM system (platform), Zebris Medical GmbH, Isny im Allgäu, Germany; Foot Print for Windows ${ }^{\circledR}$ (software), Inter Reha, Tokyo, Japan]. Subjects stood on the platform for $30 \mathrm{~s}$ in bare feet and with their vision fixed at a point $2 \mathrm{~m}$ in front of them at eye level (eyes open) or with their eyes closed. The sum of the tracks of the center of gravity is the body sway test; the extent of movement of the center of pressure directly relates to the subject's ability to maintain static balance. For the body sway test, smaller values were better in terms of functionality of the subject.

\section{Cognitive functions}

Critical flicker fusion (CFF) test This test is believed to assess the integrative capacity of the central nervous system (CNS) using TAKEI KIKI KOGYO FLICER ITEM No. 501. Subjects were required to discriminate flicker from fusion, and vice versa, of four light-emitting diodes arranged in a $1 \mathrm{~cm}$ square on a black background. Individual thresholds were determined by the psychophysical method of limits on two ascending (flicker to fusion) and two descending (fusion to flicker) scales. The mean of these two ascending and two descending presentations was used as the threshold frequency in Hz. A decreased threshold was indicative of impairment. The test has been shown to be sensitive to psychoactive compounds [23].

Simple discrimination reaction (SDR) test The SDR test is included in a Performance Test Program (NoruPro Light Systems, Inc., Tokyo, Japan). The test measures the reaction time and hand-eye coordination skills of the subjects. Subjects were required to right click on a mouse when a blue circle was lit, or left click when a white circle was lit, as quickly as possible. The mean total reaction time (s) and the rate of correct answer (\%) of 60 trials were recorded. An increase (slowing) in reaction time was indicative of impairment. The test has been shown to be sensitive to psychoactive compounds [23]. Smaller values were better, in terms of functionality of the subject for the SDR reaction time, while larger values were better for the SDR accuracy rate $(\%)$.

Recognition Memory Test (RMT) The RMT test is also included in the Performance Test Program (NoruPro Light Systems, Inc., Tokyo, Japan). Subjects were required to click the right mouse button when the same number that was displayed three times before the current one appeared, or click the left mouse button when a different number appeared. The rate (\%) of correct answers in 60 trials was recorded. A decrease in correct answers was indicative of impairment. The test has been shown to reflect the retention of short-term memory. Prior to the study, the subjects underwent an extensive training session to preclude learning effects.

\section{Subjective assessments}

The subjects' sleepiness was evaluated using the Stanford Sleepiness Scale (SSS) [24]. Alertness, well-being, and fatigue were evaluated with a visual analog scale (VAS) at 4:00 hour (Day 2) and every $2 \mathrm{~h}$ from 4:00 to 16:00 hour (i.e., 4:00, 6:00, 8:00, 10:00, 12:00, 14:00, 16:00 hour) (Day 2). The scale's extremes were 'very drowsy-very alert,' 'very bad-very good,' and 'very tired-very rested.' In terms of functionality of the subject for the SSS, smaller values were better, while larger values were better for the VAS.

In the next morning after the drug administration, the subjects were asked to fill out questionnaires of self-estimated sleep latency (SL), feeling of deep sleep, and number of wake after sleep onset (WASO).

\section{Safety}

Physical examinations, vital sign measurements, laboratory tests to ensure safety, and 12-lead electrocardiograms (ECGs) were performed pre-study and post-study. In 
addition, laboratory tests to ensure safety were performed prior to dosing, and vital signs were measured during each treatment period. Subjects were monitored for adverse experiences throughout the study. For each adverse event, the investigator indicated whether or not they thought the event was drug related. This determination was made while the investigator was blinded to the treatment.

\section{Statistical methods}

For statistical analysis, we used a two-way repeated measures ANOVA with two levels of drug (eszopiclone, placebo). After checking for interaction, we did a multiple comparison using Bonferroni for the main effects of the medicines or times. We analyzed sleep latency, feeling of deep sleep, and number of wake after sleep onset with a paired $t$ test. The statistical significance level was $p<0.05$.

\section{Results}

During this study period, no subjects dropped out from this study procedure. The results of physical functions are shown in Fig. 2, cognitive functions in Fig. 3, and subjective assessments in Table 1 and Fig. 4.
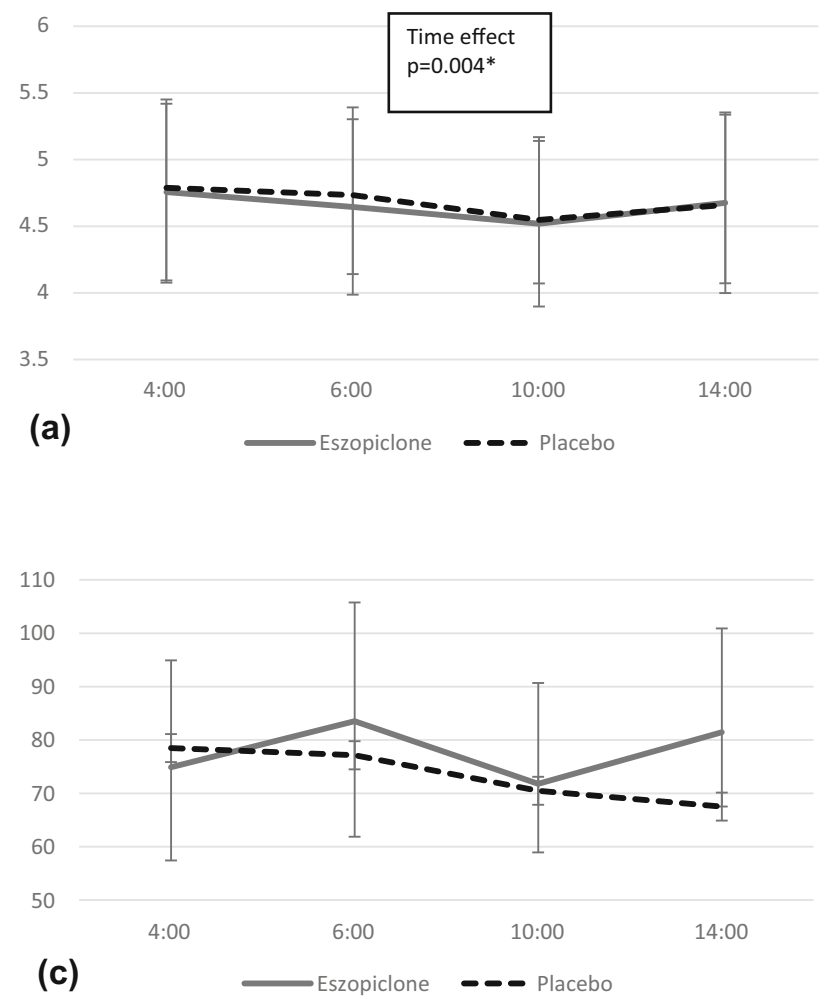

Fig. 2 Evaluations of physical functions. For the TUG, the main effects of time were seen. There were no significant differences in FRT and Body sway test between eszopiclone and placebo. a TUG,
For the TUG, the main effects of time were seen. For the $\mathrm{CFF}$, eszopiclone had significantly worse results compared to placebo in early morning (4:00 hour). There were no significant differences between eszopiclone and placebo in other objective assessments.

For the subjective scores of alertness and well-being using VAS, the main effects of time were seen. There were no significant differences between eszopiclone and placebo in SSS and fatigue using VAS. For the sleep latency, eszopiclone had significantly shorter results compared to placebo (eszopiclone vs placebo $=28.4$ vs $52.5 \mathrm{~min}$, $p=0.047$ ) (Table 1). There were no significant differences between eszopiclone and placebo in feeling of deep sleep and the number of wake after sleep onset (WASO).

\section{Discussion}

Uemura et al. [16] and Ito et al. [17] examined the effects on the next day of hypnotic to healthy young people. Zolpidem and zaleplon were reported to have significantly better results than those of placebo in CFF. However, in the present study using eszopiclone, CFF at 4:00 hour had better results than placebo. Since eszopiclone has a long half-life compered to zolpidem and zaleplon, the CFF test at 4:00 was
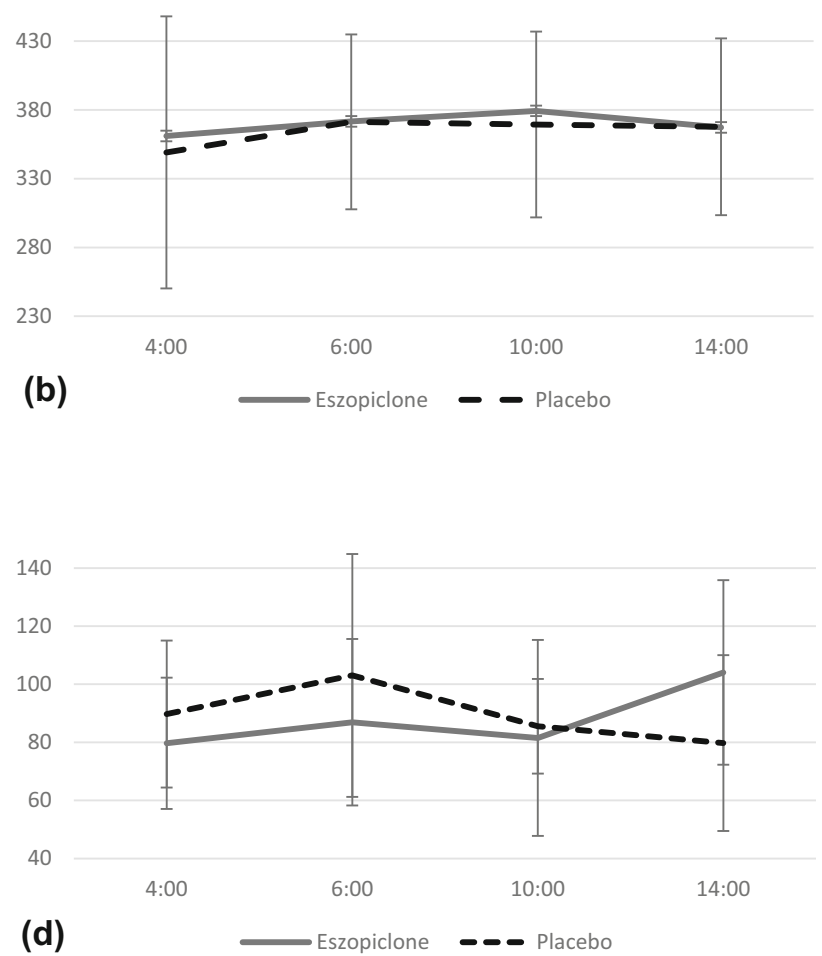

the timed up-and-go test. b FRT, functional reach test. c Body sway test (eyes open). d Body sway test (eyes closed) 

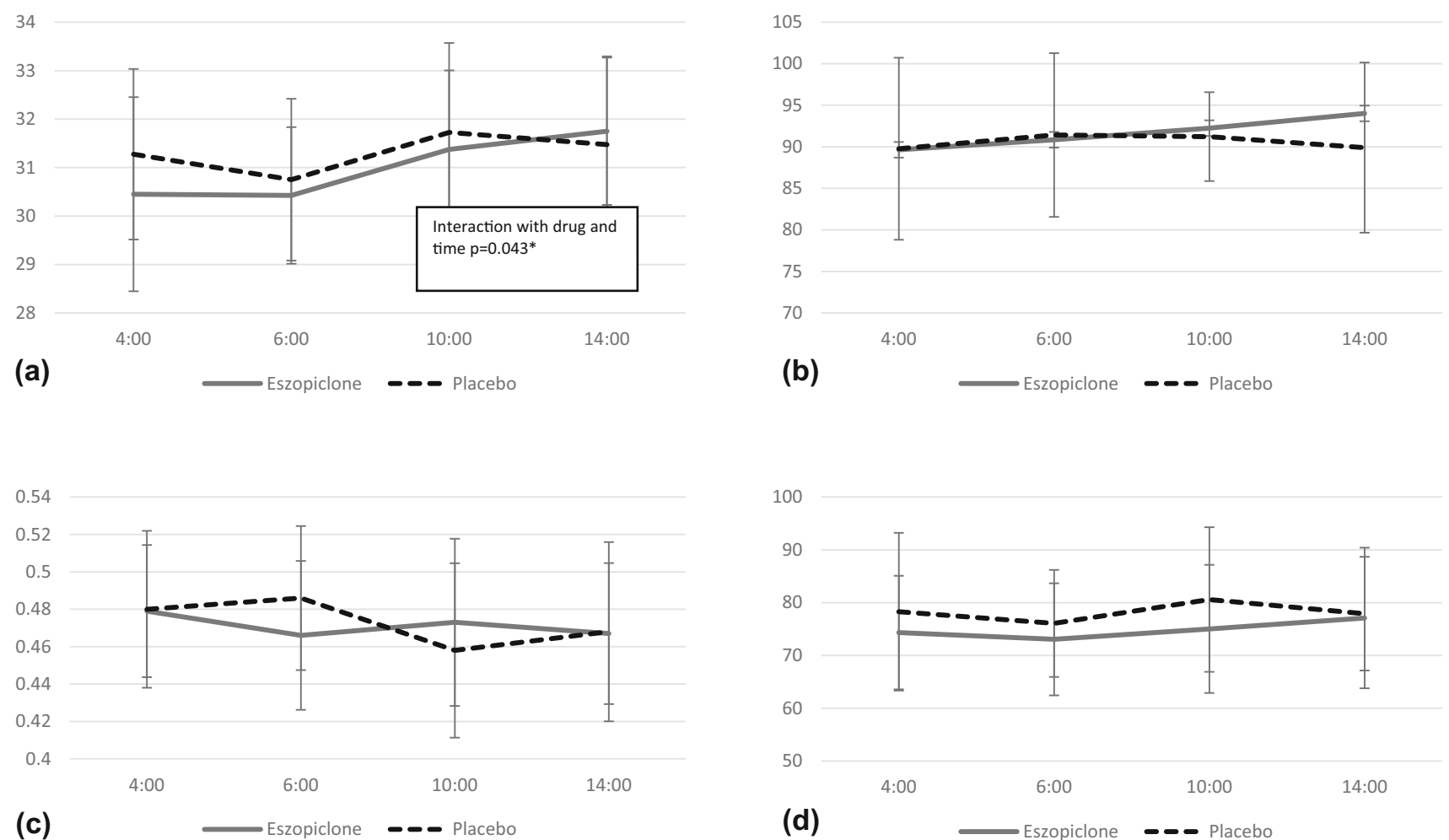

Fig. 3 Evaluations of cognitive functions. For the CFF, eszopiclone had significantly worse results compared to placebo in the early morning (4:00). There were no significant differences in STM, SDR accuracy rate, and SDR reaction time between eszopiclone and placebo. a CFF, Critical Flicker Fusion (Hz). b SDR, Simple Discrimination Reaction Accuracy Rate (\%). C SDR, Simple Discrimination Reaction Time (s). d RMT, Recognition Memory Test (\%)

Table 1 Subjective assessments of sleep parameters with eszopiclone or placebo

\begin{tabular}{|c|c|c|c|}
\hline & \multicolumn{3}{|l|}{ Sleep parameters } \\
\hline & Sleep latency (min) & Feeling of deep sleep & number of WASO \\
\hline Eszopiclone (Mean \pm SE) & $28.4 \pm 27.6$ & $2.40 \pm 1.50$ & $1.50 \pm 3.10$ \\
\hline Placebo (mean $\pm \mathrm{SE}$ ) & $52.5 \pm 38.5$ & $3.50 \pm 1.26$ & $1.80 \pm 1.61$ \\
\hline$p$ value & 0.047 & NS & NS \\
\hline
\end{tabular}

affected by the drug efficacy ( $\mathrm{t} 1 / 2$ eszopiclone $=4.8-5.1 \mathrm{~h}$, $\mathrm{t} 1 / 2$ zolpidem $=1.7-2.3 \mathrm{~h}, \mathrm{t} 1 / 2$ zaleplon $=1-1.5 \mathrm{~h}$ ) [25]. Although CFF results at 4:00 hour were worse in the subjects with eszopiclone, the other objective parameters, such as, TUG, FRT and body sway test, were the same as those with placebo; therefore the risk of falls would not be changed in these two conditions. In addition, the CFF result at 4:00 would be considered to be the main effect of eszopiclone and would contribute to the maintenance of sleep.

Uemura et al. [16] have reported that ultrashort-acting hypnotics zolpidem and triazolam did not affect cognitive motor function the next day for the elderly. This result is consistent with present study except for CFF result. Suda et al. [26] reported that administration of eszopiclone to healthy young people improved their memory function the next day; however, no significant difference was seen in our study with the elderly. This was due to the difference in the original memory functions of the young and the elderly. Although feeling of deep sleep and the number of WASO were not improved, significant shortening of sleep onset latency was observed by the administration of eszopiclone compared to that of placebo. In subjective assessments, there were no significant differences in SSS, alertness, well-being, and fatigue on the following day.

\section{Limitations}

We are aware of several limitations of this study: small sample size, only a short-term observation, and lack of objective assessment of sleep such as PSG or actigraphy. In 


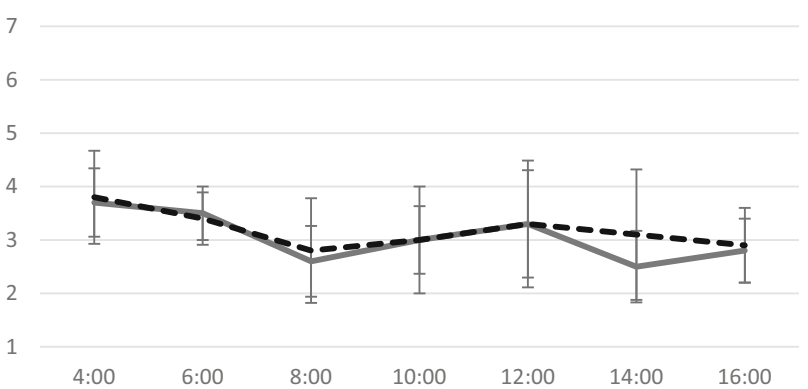

(a)
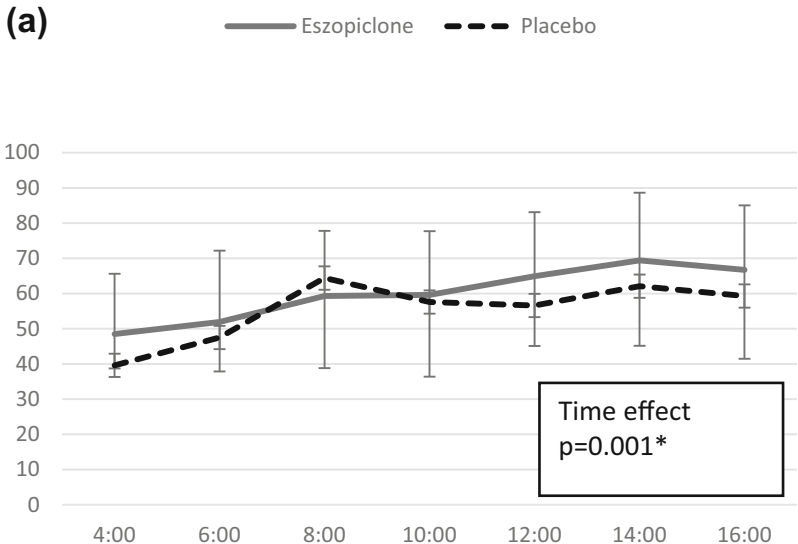

(c)

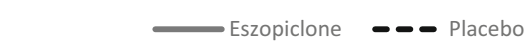

Fig. 4 Subjective assessments. For the subjective scores of alertness and well-being using VAS, the main effects of time were seen. There were no significant differences between eszopiclone and placebo in SSS and fatigue using VAS. For the sleep latency, eszopiclone had significantly shorter results compared to placebo (eszopiclone vs placebo $=28.4$ vs $52.5 \mathrm{~min}$,

addition, the examinations were conducted on the healthy elderly only at 4:00 in the early morning, and the influence on insomniacs at midnight was not considered. Therefore, these results were different from those of patients with chronic insomnia and midnight urinations. Further studies of elderly insomniacs with midnight awakenings are needed.

\section{Conclusion}

Based on the above results of the changes of physical and cognitive functions in the healthy elderly after taking hypnotics, it would be recognized that eszopiclone $1 \mathrm{mg}$ is likely to be unharmed for the healthy elderly.

Acknowledgements We would like to thank Ms. Airi Yamada for writing and editing the English for this paper.

\section{Compliance with ethical standards}

Funding There was no funding source for this study.

Conflict of interest All authors declare that he/she has no conflict of interest.
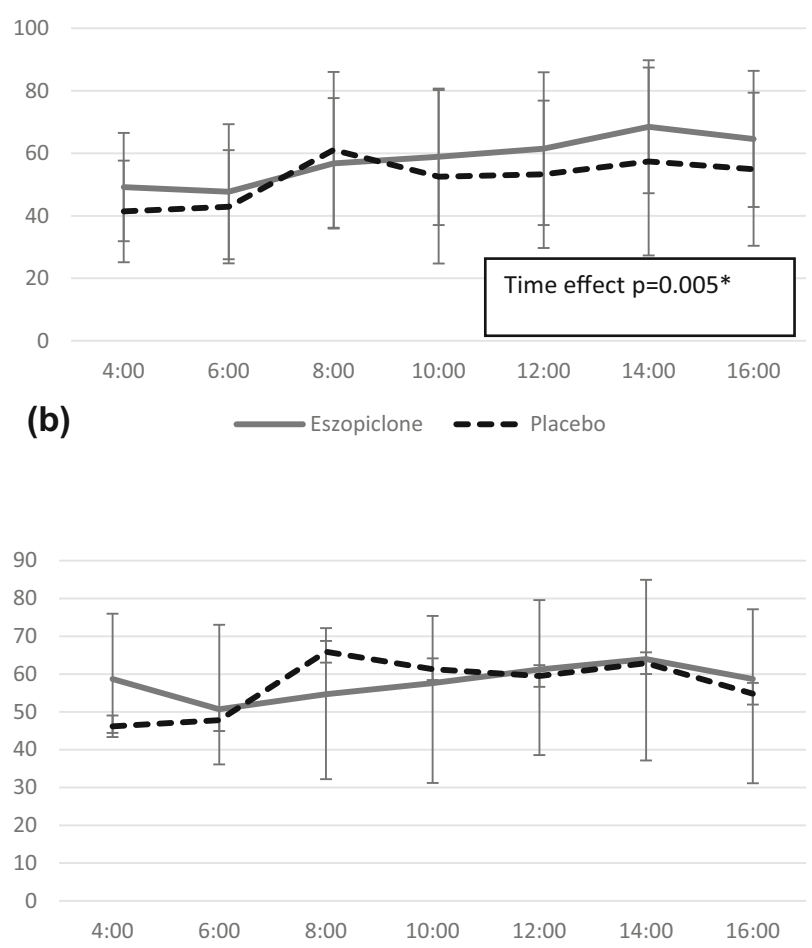

(d)

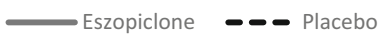

$p=0.047)$. Feeling of deep sleep and the number of wake after sleep onset (WASO) did not show any significant differences between eszopiclone and placebo. a Stanford Sleepiness Scale (SSS). b Alertness. c Well-being. d Fatigue

Ethical approval All procedures performed in studies involving human participants were in accordance with the ethical standards of the institutional and national research committee and with the 1964 Helsinki Declaration and its later amendments or comparable ethical standards.

Informed consent Informed consent was obtained from all individual participants included in the study.

Open Access This article is distributed under the terms of the Creative Commons Attribution 4.0 International License (http://crea tivecommons.org/licenses/by/4.0/), which permits unrestricted use, distribution, and reproduction in any medium, provided you give appropriate credit to the original author(s) and the source, provide a link to the Creative Commons license, and indicate if changes were made.

\section{References}

1. Ohayon MM. Epidemiology of insomnia: what we know and what we still need to learn. Sleep Med Rev. 2002;6(2):97-111.

2. Morin CM, LeBlanc M, Daley M, Gregoire JP, Mérette C. Epidemiology of insomnia: prevalence, self-help treatments, consultations, and determinants of help-seeking behaviors. Sleep Med. 2006;7(2):123-30 (Epub 2006 Feb 3).

3. Doi YMM, Okawa M, Uchiyama M. Prevalence of sleep disturbance and hypnotic medication use in relation to sociodemographic factors 
in the general Japanese adult population. $\mathrm{J}$ Epidemiol. 2000;10(2):79-86.

4. Kaneita Y, Uchiyama M, Takemura S, et al. Use of alcohol and hypnotic medication as aids to sleep among the Japanese general population. Sleep Med. 2007;8(7-8):723-32.

5. Abe S, Enomoto M, Kitamura S, Hida A, Tachimori H, Tsutsui T, Kaneita Y, Kusanagi H, Shimizu T, Mishima K. Prescription hypnotics and associated background factors in a large-scale Japanese database. Sleep Biol Rhythms. 2012;10(4):319-27.

6. Moloney ME, Konrad TR, Zimmer CR. The medicalization of sleeplessness: a public health concern. Am J Public Health. 2011;101(8):1429-33.

7. Quera-Salva MA, Orluc A, Goldenberg F, Guilleminault C. Insomnia and use of hypnotics: study of a French population. Sleep. 1991;14(5):386-91.

8. Komada Y, Nomura T, Kusumi M, Nakashima K, Okajima I, Sasai T, Inoue Y. Correlations among insomnia symptoms, sleep medication use and depressive symptoms. Psychiatry Clin Neurosci. 2011;65(1):20-9.

9. Barbone F, McMahon AD, Davey PG, et al. Association of roadtraffic accidents with benzodiazepine use. Lancet. 1998;352(9137):1331-6.

10. Verster JC, Veldhuijzen DS, Patat A, et al. Hypnotics and driving safety: meta-analyses of randomized controlled trials applying the on-the road driving test. Curr Drug Saf. 2006;1(1):63-71.

11. Verster JC, Veldhuijzen DS, Volkerts ER. Residual effects of sleep medication on driving ability. Sleep Med Rev. 2004;8(4):309-25.

12. Bauer TK, Lindenbaum K, Stroka MA, et al. Fall risk increasing drugs and injuries of the frail elderly-evidence from administrative data. Pharmacoepidemiol Drug Saf. 2012;21(12):1321-7.

13. Landi F, Onder G, Cesari M, et al. Psychotropic medications and risk for falls among community-dwelling frail older people: an observational study. J Gerontol A Biol Sci Med Sci. 2005;60(5):622-6.

14. Ito SU, Kanbayashi T, Takemura $\mathrm{T}$, et al. Acute effects of zolpidem on daytime alertness, psychomotor and physical performance. Neurosci Res. 2007;59(3):309-13.

15. Ito W, Shimizu K, Ito $S$, et al. The evaluation of physical and cognitive functions, arousal levels and moods in healthy university students after zaleplon administration. Sleep. 2010;33:A309-10.

16. Uemura SI, Kanbayashi T, Wakasa M, Satake M, Ito W, Shimizu K, Shioya T, Shimizu T, Nishino S. Residual effects of zolpidem, triazolam, rilmazafone and placebo in healthy elderly subjects: a randomized double-blind study. Sleep Med. 2015;16(11):1395-402.

17. Inomata S, Uemura SI, Wakasa M, Osawa Y, Shimizu K, Ito W, Kanbayashi T, Shimizu T. The effects of triazolam and rilmazafone on the physical and cognitive functions in healthy elderly persons. Akita J Med. 2011;38:63-9.

18. Nakajima M, Homma M, Igarashi T, et al. Survey on benzodiazepine prescriptions. J Pharm Health Care Sci. 2010;36:863-7.

19. Buscemi N, Vandermeer B, Friesen C, Bialy L, Tubman M, Ospina M, Klassen TP, Witmans M. The efficacy and safety of drug treatments for chronic insomnia in adults: a meta-analysis of RCTs. J Gen Intern Med. 2007;22(9):1335-50.

20. Huedo-Medina TB, Kirsch I, Middlemass J, Klonizakis M, Siriwardena AN. Effectiveness of non-benzodiazepine hypnotics in treatment of adult insomnia: meta-analysis of data submitted to the Food and Drug Administration. BMJ. 2012;345:e8343.

21. Podsiadlo D, Richardson S. The timed "Up \& Go": a test of basic functional mobility for frail elderly persons. J Am Geriatr Soc. 1991;39:142-8.

22. Duncan PW, Weiner DK, Chandler J, et al. Functional reach: a new clinical measure of balance. J Gerontol. 1990;45:M192-7.

23. Hindmarch I. Psychomotor function and psychoactive drugs. 1980. Br J Clin Pharmacol. 2004;58:S720-40 (discussion S41-3).

24. Hoddes E, Zarcone V, Smythe H, et al. Quantification of sleepiness: a new approach. Psychophysiology. 1973;10:431-6.

25. McCrae CS, Ross A, Stripling A, Dautovich ND. Eszopiclone for late-life insomnia. Clin Interv Aging. 2007;2(3):313-26.

26. Suda H, Uemura SI, Sagawa Y, Tokunaga J, Imanishi A, Takahashi Y, Takahashi J, Kikuchi Y, Kanbayashi T, Shimizu T. Residual effects of eszopiclone on daytime alertness, psychomotor and physical performance. SLEEP. 2014; 37 (Abstract Supplement A356). 